\title{
The Role of Neoadjuvant and Adjuvant Treatment for Adenocarcinoma of THE Upper Gastrointestinal Tract
}

\author{
C. Matuschek ${ }^{1}$, E. Bölke ${ }^{1}$, M. Peiper ${ }^{3}$, W. T. Knoefel ${ }^{4}$, W. Budach ${ }^{1}$, A. Erhardt ${ }^{5}$, A. Scherer ${ }^{6}$, P. A. Gerber ${ }^{1}$, \\ B. A. Buhren ${ }^{1}$, N. Gattermann' , S. E. Baldus ${ }^{8}$, E. Rusnak ${ }^{1}$, V. Shukla ${ }^{9}$, K. Orth ${ }^{2}$ \\ ${ }^{1}$ Klinik für Strahlentherapie und Radiologische Onkologie, Universitätsklinik, Heinrich-Heine-Universität Düsseldorf \\ ${ }^{2}$ Klinik für Allgemein, Viszeral- und Gefäßchirurgie, Klinikum Region Hannover \\ ${ }^{3}$ Klinik für Allgemein-, Viszeral- und Unfallchirurgie, Kliniken Essen-Süd, Essen \\ ${ }^{4}$ Klinik für Allgemein, Viszeral- und Kinderchirurgie, Universitätsklinik, Heinrich-Heine-Universität Düsseldorf \\ ${ }^{5}$ Klinik für Gastroenterologie, Hepatologie und Infektiologie, Universitätsklinik, Heinrich-Heine-Universität Düsseldorf \\ ${ }^{6}$ Institut für Radiologie, Universitätsklinik, Heinrich-Heine-Universität Düsseldorf \\ ${ }^{7}$ Klinik für Hämatologie, Onkologie und Klinische Immunologie, Universitätsklinik, Heinrich-Heine-Universität Düsseldorf \\ ${ }^{8}$ Institut für Pathologie, Universitätsklinik, Heinrich-Heine-Universität Düsseldorf \\ ${ }^{9}$ Methodist Radiation Therapy, Houston Texas
}

\begin{abstract}
Both locally advanced adenocarcinoma of the stomach and gastro-esophageal junction are associated with poor prognosis due to the lack of effective treatment. Recently multimodal treatment consisting of neoadjuvant chemotherapy in combination with radiotherapy is reported to improve survival when compared to surgery alone. Neoadjuvant therapy in these locally advanced tumors allows for early tumor responses and the extent of tumor regression that can be achieved is considered a significant prognostic factor. This, in turn, increases the resectability of these tumors. Also due to the high frequency of lymph node metastasis, patients with locally advanced adenocarcinoma should undergo a D2 lymphadenectomy. Postoperative chemoradiation and perioperative chemotherapy have been studied in gastric adenocarcinomas and showed a survival benefit. However, the surgical techniques used in these trials are no longer considered to be standard by today's surgical practice. In addition, there are no standard recommendations for adjuvant chemotherapy or chemoradiation after $\mathrm{R} 0$ resection and adequate lymph node dissection.
\end{abstract}

\section{INTRODUCTION}

Radiation therapy for adenocarcinoma of the upper gastrointestinal tract is reliant on both tumor location and stage. In Germany, distal esophageal adenocarcinoma of the esophagus is more common than squamous cell carcinoma of the distal esophagus and shows an over-all higher incidence. Also, the diagnosis of adenocarcinoma of the distal esophagus (Barrett's carcinoma) usually occurs in earlier stages compared to squamous cell carcinoma and therefore has an overall better long-term outcome. Although the treatment of advanced esophageal and gastric cancer includes neoadjuvant chemoradiation, neoadjuvant chemotherapy is the treatment of choice. The utilization of ra-

* This work is dedicated to Kim Speer, Thomas Speer and Roland Radner. diotherapy in the treatment of advanced gastric and lower esophageal cancer is not only underrepresented, but also less investigated in prospective studies. In this review we are providing insights into the role of radiation therapy of adenocarcinomas of the upper gastrointestinal tract.

\section{NeoAdjuVAnt Therapy of Gastro- ESOPHAGEAL JUNCTION}

Adenocarcinomas of the distal esophagus have a high propensity of lymph node metastasis and transcending mucosal spread of disease. These are the main factors limiting the curative potential of surgical treatment. Therefore, the use of multimodal therapeutic approaches in locally advanced Barrett's carcinoma (T3 and T4 stage) and in patients with extensive lymph node metastasis is indicated. The current data for postoperative radiation therapy or chemotherapy do not provide convincing evidence for either treatment.

In recent years, the efficacy of neoadjuvant therapy approaches in the treatment of esophagus tumors (e.g. adenocarcinoma and squamous cell carcinomas) has been investigated in various prospective randomized studies and metaanalyses [1-4]. Gebski et al. summarize the available randomized trials from 1982 to 2006 for neoadjuvant chemotherapy or chemoradiation [5]. In the included trials, the applied radiation doses ranged from 20-50 Gy, whereas the majority of chemotherapeutic trials utilized cisplatin and 5-fluorouracil administered either concurrently or sequentially in combination with radiotherapy. The chemoradiation of both adeno- and squamous cell carcinomas demonstrated a significant improvement $(13 \%)$ in the 2 -year overall survival rates as compared to a single modality of surgical resection (hazard ratio $0.81, \mathrm{p}=$ 0.002).

Interestingly, a significant survival advantage was demonstrated for adenocarcinomas of the distal esophagus for both neoadjuvant chemotherapy and neoadjuvant chemoradiation. However, in squamous cell carcinoma of the esophagus the survival advantage was only observed in the neoadjuvant concurrent 
chemoradiation therapy. The benefit of neoadjuvant therapy is directly correlated to the extent of the primary tumor size, whereas the treatment of locally advanced tumors with neoadjuvants revealed higher survival rates.

A recent metaanalysis reported an improved survival rate of $7 \%$ after 2 years following administration of neoadjuvant chemotherapy for esophageal cancer compared to a organ resection alone (HR 0.9, p = 0.05) [5]. However, this significant improvement could only be statistically demonstrated for adenocarcinomas of the esophagus (HR 0.78), but not for patients with squamous cell carcinomas (HR 0.88, $\mathrm{p}=0.12$ ).

The role of neoadjuvant radiotherapy was also investigated in a metaanalysis of 1147 patients [5 randomized controlled studies of potentially resectable esophageal cancer, mostly squamous cell carcinomas] [6]. After a median follow-up of 9 years, no significant survival benefit for neoadjuvant radiotherapy was noted. Therefore, at this time neoadjuvant radiotherapy cannot be recommended as a standard treatment option.

With regard to most studies regarding multimodal treatment of gastro-esophageal junction cancer or squamous cell carcinoma of the esophagus, it appears to be a major disadvantage that both tumor entities were mostly treated and analyzed in one group. Therefore, interpretations of the metaanalysis regarding tumors of the upper gastrointestinal tract are very difficult to interpret.

Although one randomized study by Walsh et al. described an advantage for the combined radiochemotherapy of adenocarcinoma, many discrepancies were reported by this study [7]. Moreover, the study was limited to a relatively small number of patients and a disproportionately poor survival rate in the surgical treatment group according to the literature. Nevertheless, one big advantage of this study is that only adenocarcinomas of the upper gastrointestinal tract were included.

Another important study is the Cross study from van der Gast, presented at the ASCO 2010 meeting [51]. In this study, 363 patients with advanced stages of esophageal cancer, high percentage of adenocarcinoma $(74 \%)$ and lymph node involvement were randomized to surgery alone or radiotherapy and chemotherapy with carboplatin / paclitaxel prior to surgery. In this study the patients benefited significantly from the neoadjuvant therapy in terms of a prolonged survival rate. Interestingly, the effect was more pronounced in the small group of squamous cell carci- nomas, while the larger group of adenocarcinomas showed only a trend. There was no increase of perioperative mortality in the neoadjuvant treatment arm.

Neoadjuvant radiotherapy is poorly evaluated on a phase III level. One randomized study from China investigated the value of preoperative radiotherapy and included 370 patients receiving $40 \mathrm{~Gy} / 2$ Gy fractions with linear arc or Co 60 [19]. An analysis of the study population showed a significant increase for the survival rate after 5 years from $20 \%$ to $30 \%(p=0.009)$ when treated with radiotherapy. The local tumor control also increased form $48 \%$ to $61 \%$.

A prospective randomized multicenter study by Stahl et al. compared adenocarcinomas of the gastroesophageal junction after neoadjuvant chemoradiation or neoadjuvant chemotherapy with regard to the survival of patients after tumor resection [8]. Patients with locally advanced (uT3-4NXM0) adenocarcinoma of the lower esophagus or gastric cardia were randomly allocated to one of two treatment groups: Induction chemotherapy (15 weeks) followed by surgery ( $\operatorname{arm~A);~}$ or chemotherapy (12 weeks) followed by chemo-radiotherapy ( 3 weeks) followed by surgery (arm B). Primary outcome was overall survival time. A total of 354 patients were needed to detect a $10 \%$ increase in 3year survival from $25 \%$ to $35 \%$ by addition of radiation therapy. The study was prematurely closed due to low accrual. The median observation time was 46 months. A total of 126 patients were randomly assigned and 119 eligible patients were evaluated. The number of patients undergoing complete tumor resection was not different between treatment groups $(69.5 \%$ vs $71.5 \%)$. Patients in arm B had a significant higher probability of showing pathologic complete response $(15.6 \%$ vs $2.0 \%)$ or tumor-free lymph nodes $(64.4 \%$ vs $37.7 \%)$ at resection. Preoperative radiation therapy improved 3-year survival rate from $27.7 \%$ to $47.4 \%$ (log-rank $\mathrm{p}=0.07$, hazard ratio adjusted for randomization strata variables $0.67,95 \% \mathrm{CI}, 0.41$ to 1.07). Postoperative mortality was not significantly increased in the chemo-radiotherapy group $(10.2 \%$ vs $3.8 \% ; \mathrm{p}=0.26)$. Although the study was closed early and statistical significance was not achieved, results point to a survival advantage for preoperative chemoradiotherapy compared with preoperative chemotherapy in adenocarcinomas of the esophago-gastric junction.

An overview of the randomized studies comparing neoadjuvant radio-chemotherapy versus surgery alone is shown in Table 1.

Table 1. Survival rate after neoadjuvant radio-chemotherapy for locally advanced adenocarcinoma.

\begin{tabular}{lcrrr}
\hline Study & $\begin{array}{c}\text { Adeno-carcinoma } \\
\mathrm{n}(\%)\end{array}$ & Survival RTX & Survival Surgery & p-value \\
\hline Walsh et al. 1996 [7] & $113(100 \%)$ & $32 \%(3 \mathrm{y})$ & $6 \%(3 \mathrm{y})$ & 0.01 \\
Urba et al. 2001 [43] & $75(75 \%)$ & $30 \%(3 \mathrm{y})$ & $16 \%(3 \mathrm{y})$ & 0.15 \\
Burmeister et al. 2005 [44] & $158(62 \%)$ & $28 \%(3 \mathrm{y})$ & $30 \%(3 \mathrm{y})$ & 0.81 \\
Tepper et al. 2008 [45] & $42(75 \%)$ & $39 \%(5 \mathrm{y})$ & $16 \%(5 \mathrm{y})$ & 0.002 \\
Stahl et al. 2009 [8] & $119(100 \%)$ & $47.7 \%(3 \mathrm{y})$ & $27.4 \%$ & 0.07 \\
& & & $(\mathrm{CHX}, 3 \mathrm{y})$ & \\
\hline
\end{tabular}

$\mathrm{RTX}=$ radio-chemotherapy, $\mathrm{CHX}=$ chemotherapy, $\mathrm{y}=$ years 
Unfortunately, there is a lack of prospective randomized phase III trials due to the low patient recruitment.

Finally, it should be noted that the MRC trial, a trial investigating the role of perioperative chemotherapy for esophageal cancer, showed a significant survival benefit for adenocarcinoma of the distal esophagus as compared to squamous cell carcinoma [9]. The new S3 guideline in Germany recommends a neoadjuvant chemo- or radio-chemotherapy for T2 tumors.

\section{NeOAdJuVAnt Chemotherapy for GASTRIC ADENOCARCINOMA}

A frequently cited multicenter trial (MAGIC trial) investigated the efficacy of a pre- and postoperative chemotherapy. This regimen consisted of three cycles of epirubicin, cisplatin and 5-FU with the endpoints of determining tumor control and survival rate in all patients with gastric adenocarcinoma ( $\mathrm{n}=372 \mathrm{pa}-$ tients) and of patients with tumors in the gastricesophageal junction ( $n=131$ patients). The combined arm showed a benefit especially for the adenocarcinoma of the distal esophagus. For patients with tumors of the gastric esophageal junction this could not be demonstrated since it would have been an unplanned subgroup analysis [10].

Unfortunately, only $42 \%$ of all patients in the chemotherapy arm finished the study according to the protocol. $34 \%$ of all patients finished their treatment after preoperative chemotherapy and surgery without receiving postoperative chemotherapy. The authors described a 5-year survival rate of $36.3 \%$ compared to $23 \%$ in favour of the chemotherapy group. Because more than half of all patients did not have a postoperative chemotherapy, the observed effect is probably an effect of the preoperative chemotherapy. This work promoted the use of neoadjuvant chemotherapy in gastric cancer (Table 2).

A comparable trial was a French multicenter study [FNLCC Accord07-FFCD 9703] [11]. In this study, $75 \%$ of all patients had adenocarcinoma of the distal esophagus or of the gastro-esophageal junction. The $\mathrm{R} 0$ resection rate after neoadjuvant therapy, which consisted of 2-3 cycles of cisplatin and 5-FU, was significantly improved compared to surgical resection alone $(84 \%$ vs $73 \%, p=0.04)$. The authors of this study observed a downsizing for lower tumor and nodal stages in the mutimodal treatment arm. Furthermore, in the multimodal treatment arm 1-4 course chemotherapy was planned as in the MAGIC trial, but only $50 \%$ of their patients were able to get the chemotherapy. The difference in the 5 year disease free survival rate was $13 \%$ (34\% vs $21 \%, \mathrm{p}=0.0033)$ and the 5 year overall survival rate was $38 \%$ vs $24 \%$ (p $=0.021$ ) in favour of the neoadjuvant therapy.

\section{Role of AdjuVANT CHEMOTHERAPY FOR THE GASTRIC ADENOCARCINOMA}

S1 compared the two arms of a Japanese trial with 530 patients treated with surgery alone and 529 patients treated with postoperative chemotherapy using Floropyrimidin and found no difference between the treatment arms [12]. Almost all patients received a D2 lymphadenectomy. S1 was able to reduce the risk for peritoneal and lymphogenic recurrences. Analysis for the 3-year survival rate revealed that adjuvant therapy was significantly associated with longer survival compared to surgery alone $(p=0.003)$. Other metaanalyses for randomized studies which analyzed postoperative chemotherapy versus surgery alone revealed a marginal survival benefit for the adjuvant therapy [1317]. Nevertheless, these results could not be repeated outside of Japan and may be restricted to the Asian region. Currently, no study exists in the Western hemisphere analyzing a sufficient number of patients revealing a significant benefit with adjuvant chemotherapy [18].

\section{NEOADJUVANT RADIOTHERAPY FOR ADENOCARCINOMA}

A meta-analysis performed in 2007, revealed a slight but significant survival benefit for patients treated with preoperative radiotherapy [20]. However, the study population was heterogeneous including intraoperative radiotherapy patients. The available data show that there might be a benefit from this treatment protocol, though the data are not valid.

\section{AdJUVANT RADIOTHERAPY FOR GASTRIC ADENOCARCINOMA}

Until now, there were no phase III trials investigating the role of neoadjuvant radio-chemotherapy in the therapy of gastric cancer. However, recently a large American trial investigated the role of adjuvant radiochemotherapy in gastric cancer [21]. In this study, patients were randomized after curative surgery to either

Table 2. Survival rate after neoadjuvant chemotherapy for advanced adenocarcinoma.

\begin{tabular}{|c|c|c|c|c|}
\hline Study & $\begin{array}{c}\text { Adeno-carcinoma } \\
\text { n }(\%)\end{array}$ & Survival CHX + Surgery & Survival Surgery & $\mathrm{p}$ - value \\
\hline MRC, 2002 [9] & $528(66 \%)$ & $\begin{array}{l}32 \% \\
(3 y)\end{array}$ & $\begin{array}{l}25 \% \\
(3 \mathrm{y})\end{array}$ & 0.02 \\
\hline MAGIC, 2006 [10] & $\begin{array}{c}503(100 \% \text { dist. } \\
\text { esophagus and stomach) }\end{array}$ & $\begin{array}{c}36.3 \% \\
(5 \mathrm{y})\end{array}$ & $\begin{array}{l}23 \% \\
(5 \mathrm{y})\end{array}$ & 0.009 \\
\hline FFCD , 2007 [11] & $\begin{array}{c}226 \text { (100\% dist. } \\
\text { esophagus and stomach) }\end{array}$ & $\begin{array}{l}38 \% \\
(5 y)\end{array}$ & $\begin{array}{l}24 \% \\
(5 \mathrm{y})\end{array}$ & 0.021 \\
\hline
\end{tabular}

$\mathrm{CHX}=$ chemotherapy, $\mathrm{y}=$ years 
radiochemotherapy with a total radiation dosage of 45 Gy and simultaneous application of 5-FU plus leucoverin $(2$ cycles) or to no adjuvant treatment at all. After three years this study demonstrates a survival benefit of $9 \%$ for patients treated with radio-chemotherapy $(50 \%$ vs $41 \%, \mathrm{p}=0.005)$. One disadvantage of this study is the inadequate number of resected lymph nodes, since only $10 \%$ of all patients received a D2 lymphadenectomy. Therefore, the conclusion of this study is not applicable to patients treated with standard resection techniques. The inadequate surgery represents the major problem of this study, since $54 \%$ of all patients did not even undergo a D1 lymph node resection $[22,23]$.

Efforts to intensify the above mentioned McDonald study [RTOG 0114] have had no positive effect for the patients [24]. In this study, two treatment arms were analyzed: Induction chemotherapy followed by simultaneous radiation compared to cisplatin and paclitaxel (PC) or cisplatin, paclitaxel and 5-FU (PCF) in the other arm. $59 \%$ of all patients treated in the PCF arm showed grade III side effects according to CTC (common toxicity criteria). Therefore, this study was stopped. Furthermore, the PC arm showed inferior disease-free survival when compared to the McDonald study [24].

Another important study is by Kim et al [46] from Korea, confirming the SWOG results for D2 lymph node resection. Their observational study suggested a clinical benefit resulting from adjuvant postoperative chemoradiation in a series of 544 patients with adenocarcinoma of the stomach after gastric resection including D2 node dissection.

A successful intensification of the chemotherapy was achieved by Kollmannsberger et al. [47]. This phase II study included weekly paclitaxel, 24-hour continuous infusion of 5-fluorouracil, folinic acid and cisplatin in patients with advanced gastric cancer. Forty-five chemotherapy-naive patients (28 male and 17 female) with a median age of 60 (range, 35-74) years were enrolled. 5 -FU $2 \mathrm{~g} / \mathrm{m}^{2}$ was given weekly over $24 \mathrm{~h}$ i.v. preceded by folinic acid $500 \mathrm{mg} / \mathrm{m}^{2}$ as a $2 \mathrm{~h}$ infusion. Paclitaxel, $175 \mathrm{mg} / \mathrm{m}^{2}$, was administered as a 3 h-infusion on days 1 and 22 and cisplatin 50 $\mathrm{mg} / \mathrm{m}^{2}$ as $1 \mathrm{~h}$ infusion on days 8 and 29 . Six weeks of therapy (days 1, 8, 15, 22, 29, 36) followed by 2 weeks rest were considered as one cycle. A median of 3 (range 1-4) cycles were administered to 45 patients assessable for response, survival and toxicity. Five patients $(11 \%)$ obtained a complete remission and $18 \mathrm{pa}-$ tients $(40 \%)$ a partial remission (ORR 51\%; 95\% Cl: $35.8-66.3 \%)$. Responses were achieved in the liver, lymph nodes, lungs and at the site of the primary tumor. Nine patients $(20 \%)$ presented with stable disease. Thirteen patients $(29 \%)$ were considered to have failed treatment, 8 patients $(18 \%)$ due to progressive disease and 5 patients $(11 \%)$ who did not receive one complete cycle of therapy due to acute non-haematologic toxicity. The median progression-free and overall survival times were 9 (range 1-36+) months and 14 (range 2-36+) months, respectively.

It has to be noted that the CRITICS study (Chemo Radiotherapy after Induction Chemo Therapy in Cancer of the Stomach) from Holland is still enrolling pa- tients. At the Netherlands Cancer Institute, a Phase III study with daily adjuvant cisplatin and capecitabine based chemo-radiotherapy has just been finished in over 120 patients with resected gastric cancer. This study demonstrated that intensive postoperative concurrent chemo-radiotherapy is feasible. Currently, a weekly cisplatin regime is evaluated as an alternative to the daily schedule. The CRITICS study is a randomized phase III trial in which all patients will receive 3 courses of ECC (epirubicin, cisplatin, capecitabine) chemotherapy and then have a D1+ gastric resection. After surgery, patients will either receive another 3 courses of ECC chemotherapy or chemo-radiotherapy (45 Gy in 25 fractions, with daily/weekly cisplatin and daily capecitabine). In order to detect a $10 \%$ benefit in overall survival, 788 patients will have to be included. Endpoints of this study are overall and disease-free survival, toxicity and quality of life. Furthermore, tissue banking and analysis (genomics, proteomics) will be part of the study. Quality assurance both in radiotherapy and surgery (Maruyama index) will be obligatory (http://www.dccg.nl/trials/critics).

\section{Response Evaluation And Response PREDICTION}

All prospective studies have shown positive tumor response to neoadjuvant therapy, which is considered as an independent prognostic factor. These patients, the so-called responders, have a significantly better prognosis than non-responders [25-27]. The selection of non-responders may play an important role in avoiding unnecessary costs and unnecessary toxic treatments with side effects. At the moment, there are no studies revealing a benefit of immediate surgical intervention improving the prognosis of non-responders. Due to a lack of randomized studies there is the hypothesis that treatment options should be selected based on response rates detected by PET-CT.

For gastric cancer tumor specific factors like p53, microsatellite instability, aberrant DNA hypermethylation and single nucleotide polymorphisms (SNP), which are related to chemotherapy response, are not known. Such tumor specific factors should be prospectively evaluated for response prediction. HER2/neu (c-erbB-2, HER2) gene amplification and protein overexpression have been associated with poor prognosis in several solid tumors, including breast and gastric cancer. Its incidence and significance in esophageal adenocarcinoma is unknown. [28-31, 54].

\section{Clinical Evaluation of Treatment RESPONSE}

For the detection of tumor regression induced by neoadjuvant therapy, endoscopy, biopsy and endosonography are image-guided procedures, which are currently available. Yet, the interpretations of the diagnostics regarding therapeutic response or non-response are difficult [33, 34]. A decision guideline is now available in the recently updated German S 3 recommendations. The major question remains the decision between surgery or radio-chemotherapy after restaging. For squamous cell carcinoma of the esopha- 
gus, Stahl et al. [48, 49] and Bedenne [50] demonstrated that responders were not associated with survival benefit after surgery compared to radio-chemotherapy alone. Nevertheless, these results could not be confirmed for adenocarcinoma of the gastric-esophageal junction.

It remains controversial if surgical resection after neoadjuvant therapy is mandatory, while a benefit could clearly be demonstrated for patients with tumor progression and development of metastatic disease and inoperability [33]. A further disadvantage of the clinical response evaluation is the time interval for surgery. In most studies, a time interval of 4-6 weeks is needed prior to surgery [32]. In patients without neoadjuvant radiation, this time span may be shorter. Usually, the blood count should be within normal limits after 3 weeks.

\section{HistopathologicAl Response}

After neoadjuvant therapy followed by tumor resection, the histopathological regression grade should be evaluated (Fig. 3), since a substantial amount of data has suggested that pathologic tumor regression following neoadjuvant therapy is an important predictor of local recurrence and long-term survival in esophageal cancer [35]. In this context, various tumor regression grading systems were established, starting with Mandard et al. in 1994 [51]. In most of the studies patients with complete tumor regression have a significantly better survival probability. In addition, patients with less than $10 \%$ vital residual tumor cells (VRTC) (so-called major histopathological response) exhibited a significantly better 3-year survival rate after neoadjuvant radio-chemotherapy. Furthermore, the lymph node status (ypN0 vs ypN1) represented a significant prognostic parameter. Summarized, a regression classification based on grade of VRTC and lymph node status led to improved survival prediction [52, 53]. With regard to gastric cancer, Becker et al. described a similar system of tumor regression [26], which applies three grades: Grade 1 , complete $(0 \%$ vital tumor cells) or subtotal tumor regression $(<10 \%$ vital tumor cells), grade 2, partial tumor regression $(10 \%-50 \%$ vital tumor cells) and grade 3 , minimal or no tumor regression ( $>50 \%$ vital tumor cells) [26]. A correlation between tumor regression grade and survival was also observed in this study.

Several investigations showed the prognostic value of tumor response in accordance to the survival date. Patients with complete tumor regression revealed the highest benefit for multimodal treatment approach [36]. Perhaps this subgroup is the best group for future adjuvant treatment modalities.

\section{Metabolic Response}

FDG-PET (fluor-18-deoxyglucose-positronemissiontomography) has proven to be a highly sensitive method to detect response after 2 weeks following chemotherapy [36-38]. The measurement of the glucose uptake of the primary tumor after induction of neoadjuvant chemotherapy compared with the standard uptake values before chemotherapy represents differentely differently if the patient was a responder or not.

The MUNICON, a phase II study, showed that an early interruption of the treatment is not a disadvantage for metabolic non responders [36, 39]. However, these data could not be confirmed by any Phase III study. Neoadjuvant chemotherapy, based on patients' response, would be a step forward for an individualized and selective approach of neoadjuvant treatment modalities. It is also the subject of ongoing and future clinical trials [36]. For gastric cancer an association between metabolic, clinical and histopathology responses was observed. Although in gastric cancer the tumor biology and prognosis for responders and non responders is different, the current available data are not sufficient to perform a clinical trial [24, 40]. Nevertheless there are new innovative individualized therapy approaches planned, especially for patients with metabolic non response [4].

\section{The Role of Technology and Side EFFECTS OF RADIOTHERAPY}

In phase II and III studies, the treatment volume included the primary tumor with a lateral safety margin of $2 \mathrm{~cm}$ to the tumor and a cranial caudal safety margin of 3-5 cm. The involvement of suspicious lymph nodes (paraesophageal, mediastinal, coeliacal, and in certain circumstances supraclavicular) is obligatory (Figs. 1-3).

Generally, the preoperative radiation dosage ranged between 36 and 45 Gy with a fractionation dose of 1.8-2.0 Gy per day. The entire treatment period is between 4 and 5 weeks. For treatment planning, lung sparing techniques as anterior posterior / posterior anterior or opposing fields should be used. Modern CT planning helps to reduce the lung mean dosage. Interesting is an investigation on 110 patients with preoperative radio-chemotherapy. The authors showed that the postoperative complication rate correlates with the mean lung dose [42]. In this study the patients received 41-50 Gy. For chemotherapy the patients received 5-FU, taxane and cisplatin. The irradiated lung volume had a positive correlation with the incidence of lung complications. At the moment, we recommend to use DDP (cis-diaminodichloroplatinum or cisplatin) and $5 \mathrm{FU}$ as a chemotherapeutic regiment outside from studies.

The most common acute side effects during radiochemotherapy is dysphagia. The major reason is the development of a mucositis of the esophageal mucous membrane. Also late side effect on the heart and lung can occur. The dosage to the spinal cord is not critical for the different neoadjuvant concepts.

The side effects on chemotherapy are different from the side effects of radiotherapy.

Severe infections due to the vulnerability of the esophageal mucous membrane can occur.

According to some authors, a contraindication for a neoadjuvant radio-chemotherapy is the possible tumor infiltration of the tracheobronchial system. Tracheobronchial fistula can occur inducing severe complications. Yet, this is discussed controversially and goes back to a time when no stents were available. 


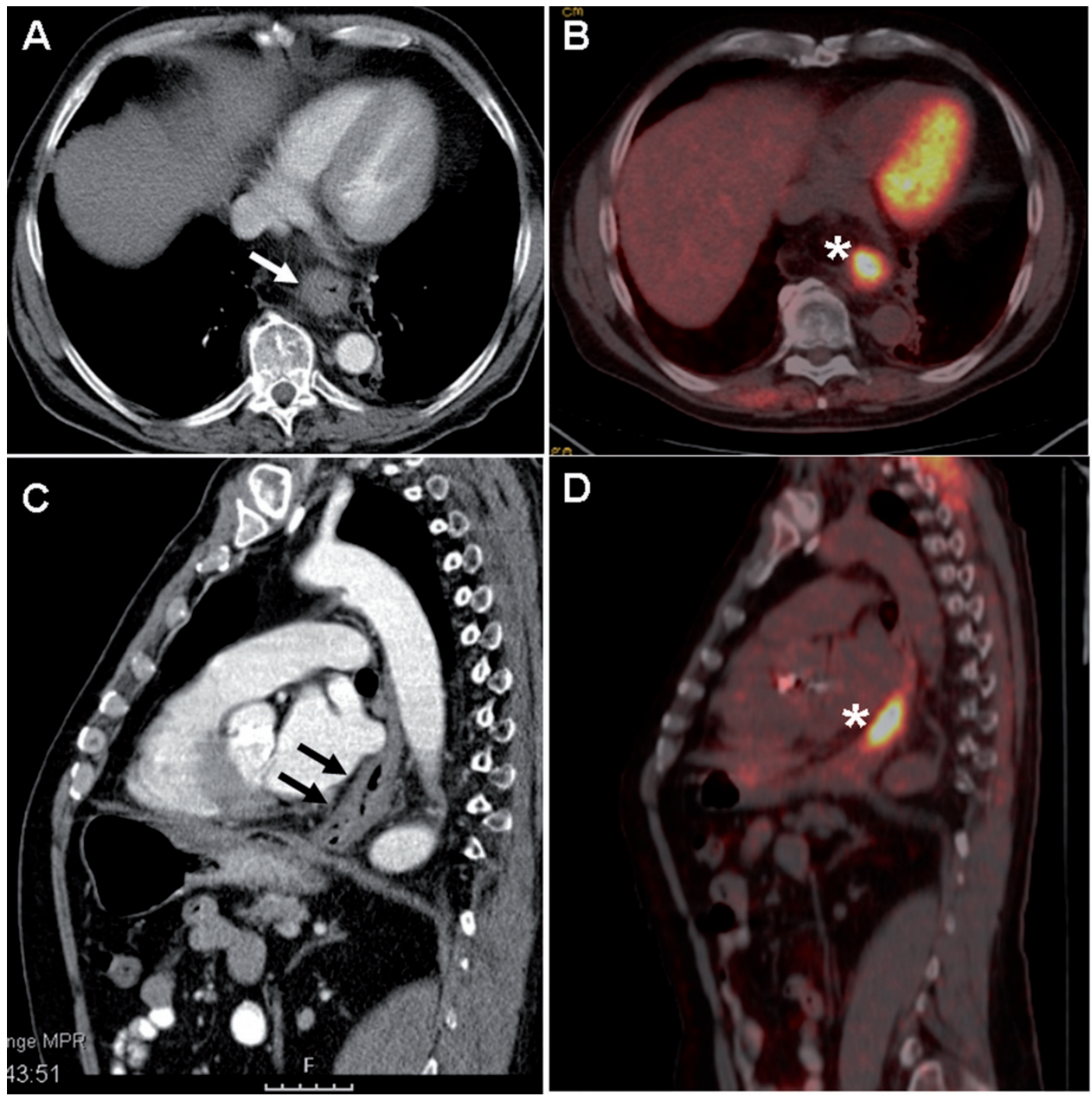

Fig. 1. PET-CT with a clear circular wall thickening of the distal esophagus and increased glucose uptake (SUV max. 12,4).

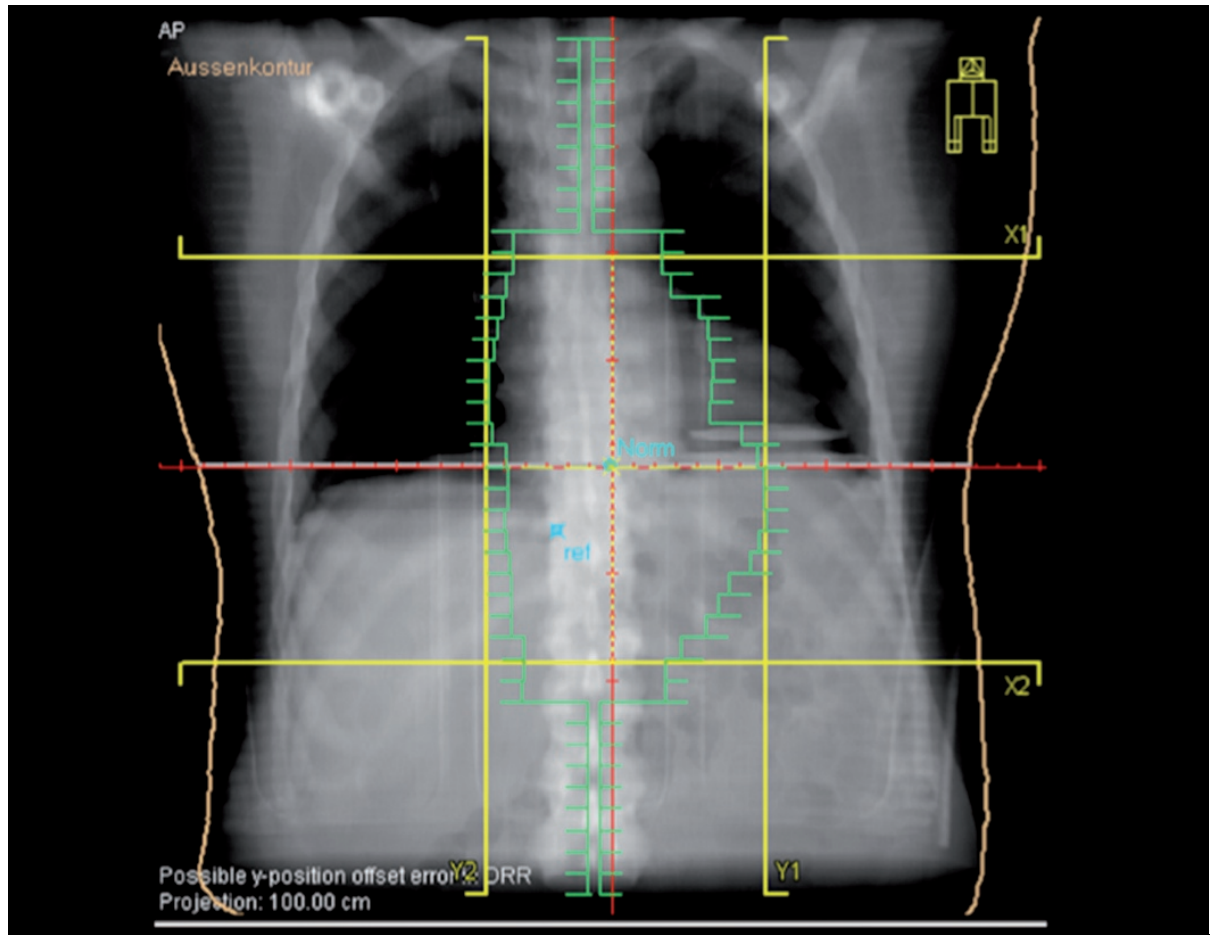

Fig. 2. Treatment plan and dose distribution for $3 \mathrm{D}$ conformal radiation therapy. 


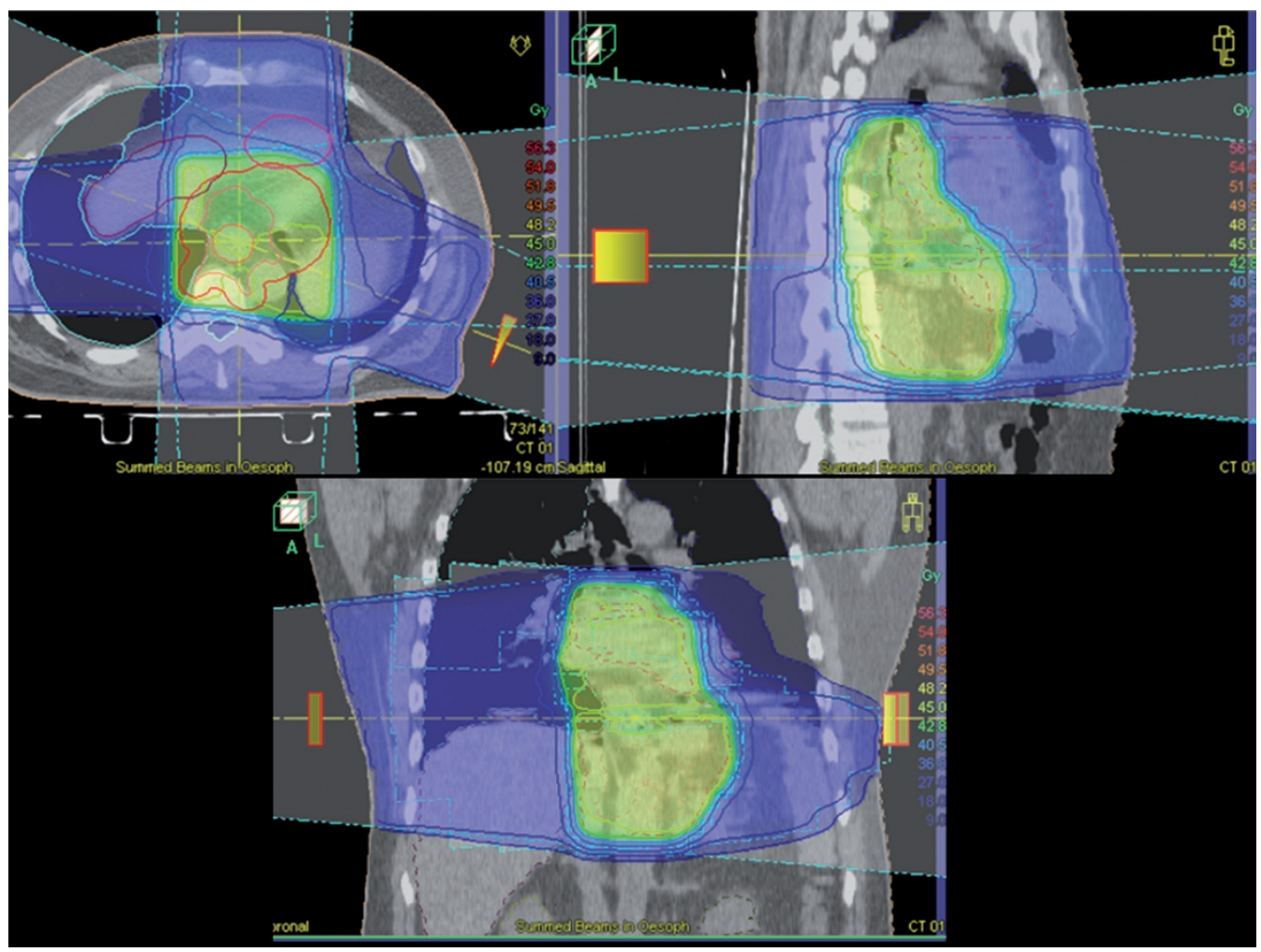

Fig. 2. Continued.
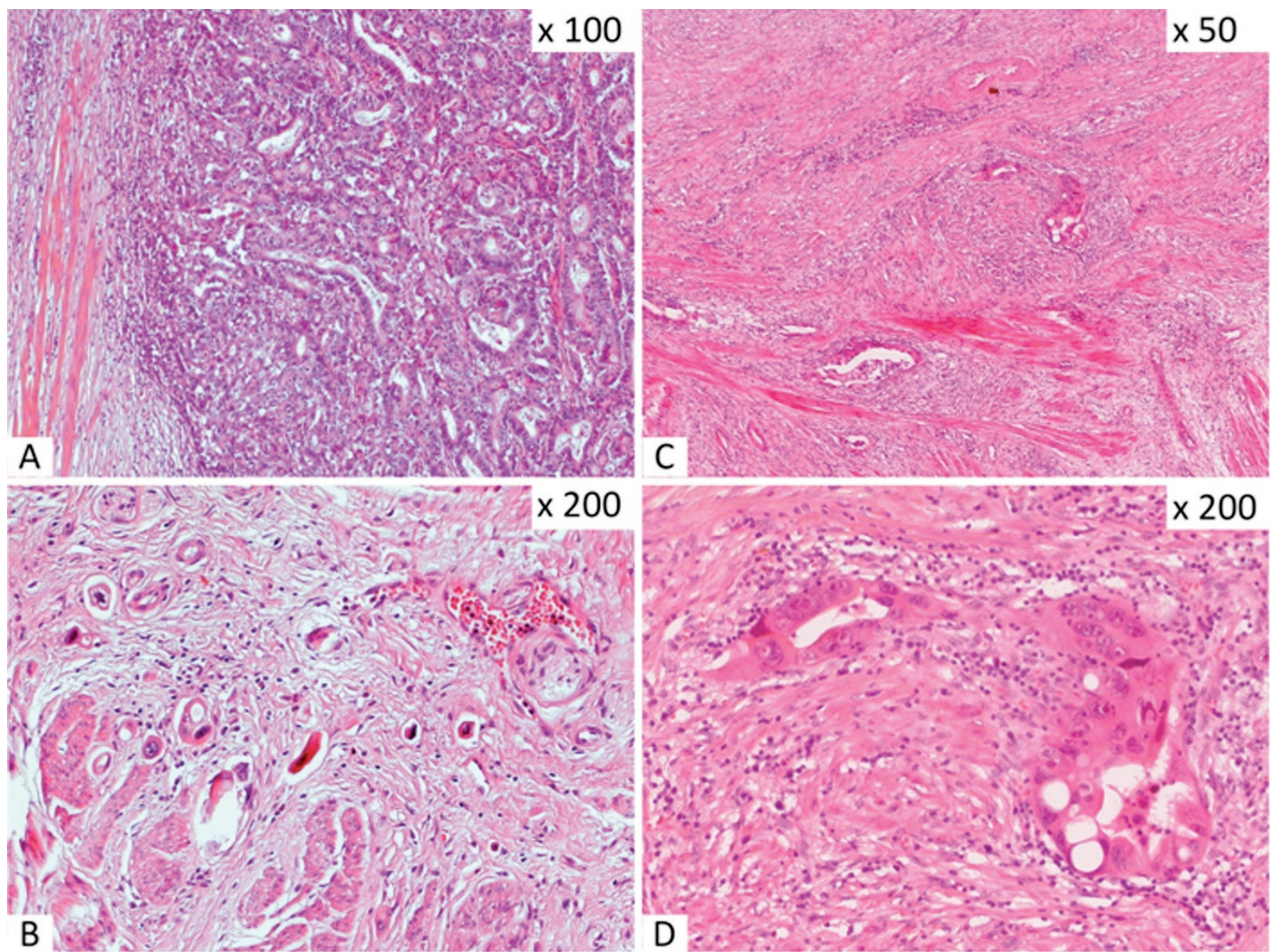

Fig. 3. Gastric adenocarcinomas after neoadjuvant chemotherapy showing no tumor regression (A) or major regression with only few scattered vital residual tumor cells (B). Adenocarcinoma of the gastro-esophageal junction exhibiting major regression after radio-chemotherapy $(\mathrm{C}, \mathrm{D})$. 
Retrospective analyses and experiences from randomized trials involving patients with squamous cell carcinoma of the esophagus showed an increase in toxicity to preoperative radiotherapy and chemo-radiotherapy resulting in an increased mortality rate. A recently published trial comparing neoadjuvant chemotherapy (PFL arm) with neoadjuvant radiochemotherapy (PLG with simultaneous 30 Gy and cisplatin etoposid) revealed no elevated mortality rate in the radio-chemotherapy arm (5 from 49 patients and 2 from 52 patients, $\mathrm{p}=0.26$ ). Length of hospital stay, intubation period, and treatment on the intensive care unit did not differ between groups [8]. Radiotherapy does not seem to be a significant risk factor. An analysis of all published randomized data shows that preoperative radio-chemotherapy in adenocarcinomas of the esophagus does not increase the postoperative mortality rate.

\section{CONCLUSION}

A meta-analysis in the treatment of advanced esophageal cancer shows a survival benefit for patients treated with neoadjuvant chemotherapy or radiochemotherapy. However, the rate of histological complete remission is higher after chemoradiation than after chemotherapy alone. After neoadjuvant therapy esophageal cancer patients with less than $10 \%$ viable residual tumor cells (high pCR rate) have a better chance of survival than patients without a significant tumor regression.

In gastric adenocarcinoma, radiotherapy has not been adequately evaluated. After a sufficient D2 lymph node resection there is no indication for adjuvant radio-chemotherapy. However, after inadequate lymph node resection, adjuvant radio-chemotherapy should be considered. The usual postoperative radiochemotherapy in the USA should not be completely transferred to European patients due to different radical surgery techniques. In experienced centers, preoperative chemoradiation is compatible with subsequent surgery and does not constitute a perioperative risk factor. Future therapies will be defined by clinical response and molecular factors and will allow for better individual therapy.

\section{REFERENCES}

1. Urschel JD, Vasan H (2003) A meta-analysis of randomized controlled trials that compared neoadjuvant chemoradiation and surgery to surgery alone for resectable esophageal cancer. Am J Surg 185(6):538-543

2. Kaklamanos IG, Walker GR, Ferry K et al (2003) Neoadjuvant treatment for resectable cancer of the esophagus and the gastroesophageal junction: a meta-analysis of randomized clinical trials. Ann Surg Oncol 10(7):754-761

3. Fiorica F, Di Bona D, Schepis F et al (2004) Preoperative chemoradiotherapy for oesophageal cancer: a systematic review and meta-analysis. Gut 53(7):925-930

4. Geh JI, Bond SJ, Bentzen SM, Glynne-Jones R (2006) Systematic overview of preoperative (neoadjuvant) chemoradiotherapy trials in oesophageal cancer: evidence of a radiation and chemotherapy dose response. Radiother Oncol 78(3):236-244

5. Gebski V, Burmeister B, Smithers BM et al (2007) Sur- vival benefits from neoadjuvant chemoradiotherapy or chemotherapy in oesophageal carcinoma: a meta-analysis. Lancet Oncol 8(3):226-234

6. Arnott SJ, Duncan W, Gignoux M et al (2005) Preoperative radiotherapy for esophageal carcinoma. Cochrane Database Syst Rev (4):CD001799

7. Walsh TN, Noonan N, Hollywood D et al (1996) A comparison of multimodal therapy and surgery for esophageal adeno-carcinoma. N Engl J Med 335(7):462-467

8. Stahl M, Walz MK, Stuschke M et al (2009) Phase III comparison of preoperative chemotherapy compared with chemoradiotherapy in patients with lo $\neg$ cally advanced adeno-carcinoma of the esophagogastric junction. $\mathrm{J}$ Clin Oncol 27(6):851-856

9. Medical Research Council Oesophageal Cancer Working Group (2002) Surgical resection with or without preoperative chemotherapy in oesophageal cancer: a randomised controlled trial. Lancet 359:1727-1733

10. Cunningham D, Allum WH, Stenning SP et al (2006) MAGIC Trial Participants. Perioperative chemo $\neg$ therapy versus surgery alone for resectable gastroesophageal cancer. N Engl J Med 355:11-20

11. Boige V, Pignon JP, Saint-Aubert B, et al. Final results of a randomized trial comparing preoperative 5-fluorouracil $(\mathrm{F})$ cisplatin $(\mathrm{P})$ to surgery alone in adeno-carcinoma of stomach and lower esophagus (ASLE): FNLCC Accord 07-FFCD 9703 trial. J Clin Oncol 2007, 25: 4510 (Abstr)

12. Sakuramoto S, Sasako M, Yamaguchi T, et al. Adjuvant chemotherapy for gastric cancer with S-1, an oral fluoropyrimidine. N Engl J Med 2007, 357: 1810-1820

13. Earle CC, Maroun JA. Adjuvant chemotherapy after curative resection for gastric cancer in non-Asian patients: revisiting a meta-analysis of randomized trials. Eur J Cancer 1999 35: 1059-1064

14. Mari E, Floriani I, Tinazzi A, et al. Efficacy of adjuvant chemotherapy after curative resection for gastric cancer: a meta-analysis of published randomized trials. A study of the GISCAD Ann Oncol 2000, 11: 837-843

15. Janunger KG, Hafström L, Glimelius B. Chemotherapy in gastric cancer: a review and updated meta-analysis. Eur J Surg Oncol 2002, 168: 597-608

16. Buyse M, Pignon J. Meta-analysis of randomized trials assessing the interest of postoperative chemotherapy and prognostic factors in gastric cancer. J Clin Oncol 2009, 25:211

17. Macdonald JS, Smalley SR, Benedetti J, et al. Chemoradiotherapie after surgery compared with surgery alone for adeno-carcinoma of the stomach or gastroesophageal junction. N Engl J Med 2001, 345: 725-730

18. Hermans J, Bonenkamp JJ, Boon MC et al (1993) Adjuvant therapy after curative resection for gastric cancer: meta-analysis of randomized trials. J Clin Oncol 11(8):1441-1447

19. Zhang ZX, Gu XZ, Yin WB et al (1998) Randomized clinical trial on the combination of preoperative irradiation and surgery in the treatment of adeno-carcinoma of gastric cardia (AGC) - report on 370 patients. Int J Radiat Oncol Biol Phys 42(5):929-934

20. Fiorica F, Cartei F, Enea M et al (2007) The impact of radiotherapy on survival in resectable gastric carcinoma: a meta-analysis of literature data. Cancer Treat Rev 33(8): $729-740$

21. Macdonald JS, Smalley SR, Benedetti J et al (2001) Chemoradiotherapy after surgery compared with surgery alone for adeno-carcinoma of the stomach or gastroesophageal junction. N Engl J Med 345(10):725730

22. Bajetta E, Buzzoni R, Mariani L et al (2002) Adjuvant chemotherapy in gastric cancer: 5 -year results of a randomised study by the Italian Trials in Medical Oncology (ITMO) Group. Ann Oncol 13(2):299-307 
23. Bouché O, Ychou M, Burtin P et al (2005) Adjuvant chemotherapy with 5-fluorouracil and cisplatin compared with surgery alone for gastric cancer: 7-year results of the FFCD randomized phase III trial (8801). Ann Oncol 16(9):1488-1497

24. Schwartz GK, Winter K, Minsky BD, et al. Randomized phase II trial evaluating two paclitaxel and cisplatin-containing chemoradiation regiments as adjuvant therapy in resected gastric cancer (RTOG-0114). J Clin Oncol 2009, 27: 1956-1962

25. Ott K, Herrmann K, Lordick F, Wieder H et al (2008). Early metabolic response evaluation by fluorine-18 fluorodeoxyglucose positron emission tomography allows in vivo testing of chemosensitivity in gastric cancer: long term results of a prospective study. Clin Cancer Res 14: 2012-2018

26. Becker K, Mueller JD, Schulmacher C, Ott K et al (2003) Histomorphology and grading of regression in gastric carcinoma treated with neoadjuvant chemotherapy. Cancer 98:1521-1530

27. Lowy AM, Mansfield PF, Leach SD, Pazdur R et al (1999) Response to neoadjuvant chemotherapy best predicts survival after curative resection of gastric cancer. Ann Surg 229:303-308

28. Napieralski R, Ott K, Kremer M, Becker K et al (2007) Methylation of tumor-related genes in neoadjuvant-treated gastric cancer: Relation to therapy response and clinicopathologic and molecular features. Clin Cancer Res 13:5095-5102

29. Ott K, Vogelsang H, Marton N, Becker K et al (2006) The thymidylate synthase tandem repeat promoter polymorphism: A predictor for tumor-related survival in neoadjuvant treated locally advanced gastric cancer. Int J Cancer 119:2885-2894

30. Ott K, Vogelsang H, Mueller J, Becker K et al (2003) Chromosomal instability rather than p53 mutation is associated with response to neoadjuvant cisplatin-based chemotherapy in gastric carcinoma. Clin Cancer Res 9:2307-2315

31. Brücher BL, Swisher SG, Königsrainer A et al (2009) Response to preoperative therapy in upper gastrointestinal cancers. Ann Surg Oncol 16:878-886

32. Beer AJ, Wieder HA, Lordick F, Ott K et al (2006) Adeno-carcinomas of esophagogastric junction: multi-detector row CT to evaluate early response to neoadjuvant chemotherapy. Radiology 239:472-480

33. Lordick F, Stein HJ, Peschel C, Siewert JR (2004) Neoadjuvant therapy for oesophagogastric cancer. Br J Surg 91:540-551

34. Jouve J, Michel P, Mariette C et al (2008) Fédération Francophone de Cancérologie Digestive. Outcome of the nonrandomized patients in the FF CD 9102 trial: Chemoradiation followed by surgery compared with chemoradiation alone in squa $\urcorner$ mous cancer of the esophagus. J Clin Oncol 26 [Suppl]:4555 (Abstr)

35. Chang F, Deere H, Mahadeva U, George S (2008) Histopathologic examination and reporting of esophageal carcinomas following preoperative neoadjuvant therapy: practical guidelines and current issues. Am J Clin Pathol 129(2):252-262

36. Ott K, Weber WA, Lordick F, Becker K et al (2006) Metabolic imaging predicts response, survival, and recurrence in adeno-carcinomas of the esophago gastric junction. J Clin Oncol 24:4692-4698

37. Weber WA, Ott K, Becker K et al (2001) Prediction of response to preoperative chemotherapy in adeno-carcinomas of the esophagogastric junction by metabolic imaging. J Clin Oncol 19(12):3058-3065

38. Wieder HA, Beer AJ, Lordick F et al (2005) Comparison of changes in tumor metabolic activity and tumor size during chemotherapy of adeno-carcinomas of the esophagogastric junction. J Nucl Med 46:2029-2034
39. Lordick F, Ott K, Krause BJ, Weber WA et al (2007) PET to assess early metabolic response and to guide treatment of adeno-carcinoma of the oesophagogastric junction: the MUNICON phase II trial. Lancet Oncol 8:797-805

40. Ott K, Fink U, Becker K, Stahl A et al (2003) Prediction of response to preoperative chemotherapy in gastric carcinoma by metabolic imaging: results of a prospective trial. J Clin Oncol 21:4604-4610

41. Lordick F, Ruers T, Aust DE et al (2008) European Organisation of Research and Treatment of Cancer (EORTC) Gastrointestinal Group: Workshop on the role of metabolic imaging in the neoadjuvant treatment of gastrointestinal cancer. Eur J Cancer 44:1807-1819

42. Wang SL, Liao Z, Vaporciyan AA et al (2006) Investigation of clinical and dosimetric factors associated with postoperative pulmonary complications in esophageal cancer patients treated with concurrent chemoradiotherapy followed by surgery. Int J Radiat Oncol Biol Phys 64(3):692-699

43. Urba SG, Orringer MB, Turrisi A et al (2001) Randomized trial of preoperative chemoradiation versus surgery alone in patients with locoregional esophageal carcinoma. J Clin Oncol 19(2):305-313

44. Burmeister BH, Smithers BM, Gebski V et al (2005) Surgery alone versus chemoradiotherapy followed by surgery for resectable cancer of the oesophagus: a randomised controlled phase III trial. Lancet Oncol 6(9): 659-668

45. Tepper JE, Krasna M, Niedzwiecki D et al (2006) Superiority of trimodality therapy to surgery alone in esophageal cancer: Results of CALGB 9781. J Clin Oncol (Meeting Abstracts) 24 [Suppl 18]:4012

46. Kim S, Lim DH, Lee J, Kang WK, MacDonald JS, Park CH, Park SH, Lee SH, Kim K, Park JO, Kim WS, Jung CW, Park YS, Im YH, Sohn TS, Noh JH, Heo JS, Kim YI, Park CK, Park K. (2005) An observational study suggesting clinical benefit for adjuvant postoperative chemoradiation in a population of over 500 cases after gastric resection with D2 nodal dissection for adenocarcinoma of the stomach. Int J Radiat Oncol Biol Phys. 63(5):1279-85.

47. Kollmannsberger C, Quietzsch D, Haag C, Lingenfelser T, Schroeder M, Hartmann JT, Baronius W, Hempel V, Clemens M, Kanz L, Bokemeyer C (2000). A phase II study of paclitaxel, weekly, 24-hour continous infusion 5fluorouracil, folinic acid and cisplatin in patients with advanced gastric cancer. Br J Cancer. 2000 83(4):458-62.

48. Stahl M, Budach W, Meyer HJ, Cervantes A; ESMO Guidelines Working Group. Esophageal cancer: Clinical Practice Guidelines for diagnosis, treatment and followup. Ann Oncol. 2010 May;21 Suppl 5:v46-9.

49. Stahl M, Stuschke M, Lehmann N, Meyer HJ, Walz MK, Seeber S, Klump B, Budach W, Teichmann R, Schmitt M, Schmitt G, Franke C, Wilke H. Chemoradiation with and without surgery in patients with locally advanced squamous cell carcinoma of the esophagus. J Clin Oncol. 2005 ;23(10):2310-7. Erratum in: J Clin Oncol. 2006 24(3):531.

50. Bedenne L, Michel P, Bouché O, Milan C, Mariette C, Conroy T, Pezet D, Roullet B, Seitz JF, Herr JP, Paillot B, Arveux P, Bonnetain F, Binquet C. Chemoradiation followed by surgery compared with chemoradiation alone in squamous cancer of the esophagus: FFCD 9102. J Clin Oncol. 2007;25(10):1160-8.

51. Mandard AM, Dalibard F, Mandard JC et al. (1994) Pathologic assessment of tumor regression after preoperative chemoradiotherapy of esophageal carcinoma; clinicopathologic correlations. Cancer 73, 2680-2686

52. Baldus SE, Mönig SP, Schröder W et al. (2004) Regression von Ösophaguskarzinomen nach neoadjuvanter Radiochemotherapie. Pathologe 25: 421-427 
53. Schneider PM, Baldus SE, Metzger R et al. (2005) Histomorphologic tumor regression and lymph node metastases determine prognosis following neoadjuvant radiochemotherapy for esophageal cancer. Ann Surg 242, 684-692

54. Thompson SK, Sullivan TR, Davies R, Ruszkiewicz AR. HER-2/neu Gene Amplification in Esophageal Adenocarcinoma and Its Influence on Survival. Ann Surg Oncol. 2011 Jan 26. [Epub ahead of print]

55. Van der Gast, Effect of preoperative concurrent chemoradiotherapy on survival of patients with resectable esophageal or esophagogastric junction cancer: Results from a multicenter randomized phase III study Abstract \#4004, ASC0 2010 Annual Meeting, 4-8 June, Chicago
Received: March 2, 2011 / Accepted: April 1, 2011

Address for correspondence:

PD Dr. med. Edwin Bölke

Klinik für Strahlentherapie und Radiologische Onkologie Heinrich-Heine-Universität Düsseldorf

Moorenstraße 5

40225 Düsseldorf

Germany

Tel.: +49-211-8117990

Fax: +49-211-81118051

Email: boelke@med.uni-duesseldorf.de 